\title{
Treatment of $o$-alkynylphenylsydnones with acids: a new route to 3-arylcinnolines
}

\author{
Andrew J. Weisner and Kenneth Turnbull* \\ Department of Chemistry, Wright State University, 3640 Colonel Glenn Hwy, \\ Dayton, $\mathrm{OH} 45435$, USA \\ E-mail: kenneth.turnbull@wright.edu
}

DOI: http://dx.doi.org/10.3998/ark.5550190.p008.474

\begin{abstract}
Treatment of $o$-alkynylphenylsydnones with triflic acid or trifluoroacetic acid provides a novel avenue to 3-arylcinnolines. With triflic acid the best results were obtained at room temperature with alkynylsydnones containing an electron-withdrawing group (or an $\mathrm{H}$ ) whereas with TFA an electron-donating group and reflux conditions were required.
\end{abstract}

Keywords: Cinnolines, fused-ring sydnones, acid-induced ring cleavage

\section{Introduction}

Sydnones are dipolar, nonbenzenoid aromatic compounds, which are the most studied members of the class of heterocycles known as mesoionic. ${ }^{1}$ Since their first preparation in $1935{ }^{2}$ on account of their intriguing structural, chemical and biological properties, ${ }^{1}$ a large number of sydnones have been reported. The most common avenue to modification of their properties has been via substitution at the C-4 position and this has been achieved by electrophilic aromatic substitution, ${ }^{3}$ lithiation ${ }^{4,5}$ or other metallation ${ }^{6,7}$ followed by reaction with an electrophile.

For some years, we have been interested in the preparation of fused-ring sydnones such as 2 with a view to exploring the modification of sydnone properties thus engendered. ${ }^{8,9}$ In this regard, it has been speculated by Coburn and O'Donnell that, in fused-ring sydnones, the stabilization achieved by the additional conjugation from the sydnone ring "might be of practical significance, since many of the simple sydnones with potentially useful biological activities lack thermal stability and frequently darken upon exposure to light and air". ${ }^{10}$ While, previously, sydno[3,4- $a]$ quinoxalines were prepared by Coburn and O'Donnell using a lithiation protocol, ${ }^{10}$ for us the most effective, general strategy to fused-ring sydnones has been to generate an electrophilic side-chain that can be trapped by the heteroaromatic sydnone ring. In this fashion, we have synthesized a sydno[4,3-c]benzotriazine, ${ }^{9}$ several 4-arylaminosydno[3,4- $a$ ]quinoxalines, $^{8}$ and a limited number of 5-substituted sydno[3,4-a]quinazolines. ${ }^{11,12}$ In order to 
explore further this concept, and extend the limited range of fused-ring sydnones available, we elected to examine the reactions of $o$-alkynylphenylsydnones 1 (prepared by Sonogashira coupling with $o$-iodophenylsydnone $)^{13}$ with various acids in the expectation that sydnoquinoline derivatives 2 would result. The present manuscript reports our findings from this study.

\section{Results and Discussion}

Our first effort to effect cyclization of the sydnone alkynes $\mathbf{1}$ to the desired sydnoquinolines 2 was to examine the efficacy of concentrated sulfuric acid in this regard. While reaction of $\mathbf{1 a}$ with sulfuric acid did lead to the corresponding fused-ring sydnone $\mathbf{2 a}$ (Table 1, entry 1) the extremely low yield led us to consider alternative avenues. We postulated that this issue could be alleviated by the use of an organic acid and, accordingly, we explored the use of trifluoromethanesulfonic acid (TfOH) and trifluoroacetic acid (TFA) as acid replacements. Unexpectedly, treatment of alkynylsydnones 1 with TfOH or TFA resulted in the isolation of 3-arylcinnolines 3 in $60-86 \%$ yield. With $\mathrm{TfOH}$, the reaction was effected in 15 minutes at room temperature and the best results were obtained with those alkynylsydnones containing electron-withdrawing moieties (or an $\mathrm{H}$ ) attached to the alkynyl aryl group (1a-c, entries 2-4). In contrast, TFA, the weaker acid, required reflux conditions for 3-6 hours and a donating group attached to the alkynyl aryl group (1d-f, entries 5-7) to effect the transformation.
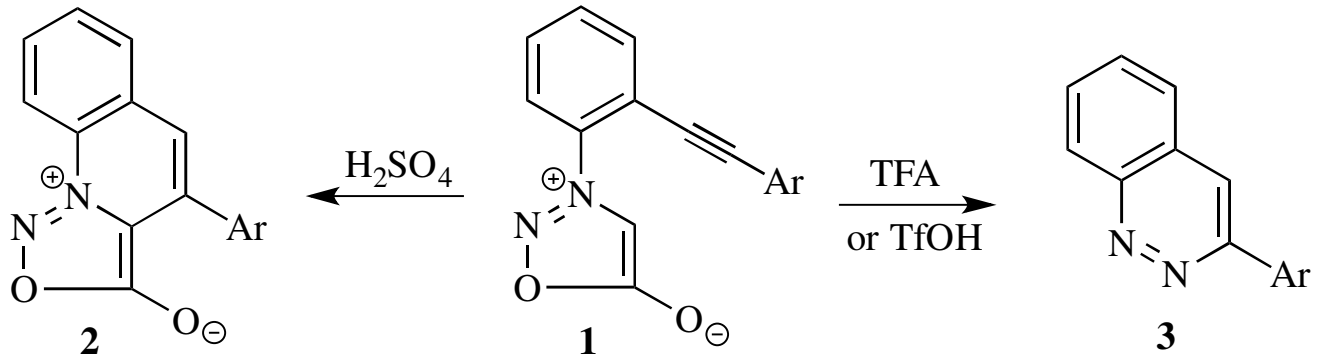

\section{Scheme 1}

Table 1. Reactions of $o$-alkynylphenylsydnones 1 with $\mathrm{H}_{2} \mathrm{SO}_{4}$, TFA or TfOH

\begin{tabular}{cccccccc}
\hline Entry & Reactant & $\mathrm{Ar}$ & Acid & Product & Yield (\%) & $\mathrm{Mp}{ }^{\circ} \mathrm{C}$ & Lit. Mp ${ }^{\circ} \mathrm{C}$ \\
\hline 1 & $\mathbf{1 a}$ & $\mathrm{Ph}$ & $\mathrm{H}_{2} \mathrm{SO}_{4}$ & $\mathbf{2 a}$ & 22 & $244-6$ & - \\
2 & $\mathbf{1 a}$ & $\mathrm{Ph}$ & $\mathrm{TfOH}$ & $\mathbf{3 a}$ & 72 & $115-6$ & $118.5-19^{14}$ \\
3 & $\mathbf{1 b}$ & $4-\mathrm{BrC}_{6} \mathrm{H}_{4}$ & $\mathrm{TfOH}$ & $\mathbf{3 b}$ & 60 & $141-2$ & Ref. $^{15}$ \\
4 & $\mathbf{1 c}$ & $4-\mathrm{ClC}_{6} \mathrm{H}_{4}$ & $\mathrm{TfOH}$ & $\mathbf{3 c}$ & 69 & $142-3$ & $146.5-8^{16}$ \\
5 & $\mathbf{1 d}$ & $4-\mathrm{MeOC}_{6} \mathrm{H}_{4}$ & $\mathrm{TFA}$ & $\mathbf{3 d}$ & 82 & $104-5$ & $105-6^{17}$ \\
6 & $\mathbf{1 e}$ & $4-\mathrm{MeC}_{6} \mathrm{H}_{4}$ & $\mathrm{TFA}$ & $\mathbf{3 e}$ & 86 & $121-2$ & $101-3^{17}$ \\
7 & $\mathbf{1 f}$ & $4-\mathrm{PnC}_{6} \mathrm{H}_{4}$ & $\mathrm{TFA}$ & $\mathbf{3 f}$ & 77 & $71-2$ & - \\
\hline
\end{tabular}


Undoubtedly, the mechanism of the reaction involves hydrolysis of the sydnone ring to a hydrazine, acid-catalyzed cyclization of the latter to the alkyne and subsequent oxidation in air. Acid-induced hydrazine formation from sydnones has been known since 1935, ${ }^{2}$ however, the utility of ortho-substituted arylsydnones as masked hydrazines for heterocycle formation has been utilized only infrequently. Indeed, to the best of our knowledge, apart from our study of such transformations in $1992,{ }^{18}$ there has been no systematic examination of this concept. The present findings exhibit further the utility of stable, crystalline $o$-substituted arylsydnones as valuable precursors to useful heterocycles and, given the rather efficient avenues to the alkynylsydnone precursors, ${ }^{13}$ may find considerable utility.

Substituted cinnolines often exhibit interesting bioactivity ${ }^{19}$ and hitherto have been prepared by a variety of methods, primary among which are intramolecular cyclizations such as the von Richter synthesis (cyclization of an arenediazonium species onto an $o$-alkyne) ${ }^{20}$ and avenues involving aryl hydrazines, ${ }^{21}$ aryl hydrazones ${ }^{22,23}$ and nitriles. ${ }^{24}$ More recently, valuable extensions to these routes have been developed using copper-catalyzed coupling protocols. ${ }^{15,17}$ In general, 3-arylcinnolines have been prepared using one or other of the aforementioned approaches ${ }^{16,17}$ and methods for the synthesis of cinnolines, in general, have been reviewed. ${ }^{25}$

\section{Conclusions}

A convenient synthesis of 3-arylcinnolines $\mathbf{3}$ from $o$-alkynyl sydnones $\mathbf{1}$ has been developed using either treatment with trifluoroacetic acid or triflic acid, depending on the nature of the alkynyl sydnone employed. A novel fused-ring sydnone $\mathbf{2 a}$ was obtained in low yield from one alkynylsydnone 1a. It is planned to examine further both synthetic avenues (to $\mathbf{2}$ or $\mathbf{3}$ ) in order to better delineate the scope and limitations of the procedures.

\section{Experimental Section}

General. The $o$-alkynylphenylsydnone 1 starting materials were prepared as reported previously. ${ }^{13}$ Melting points were determined on a Mel-Temp melting point device and remain uncorrected. Infrared spectra were performed on a $\mathrm{KBr}$ salt plate and were taken on a Nicolet 6700 FTIR. NMR spectra were obtained on a Bruker Advance $300 \mathrm{MHz} \mathrm{NMR} \mathrm{in} \mathrm{CDCl}_{3}$ and are reported relative to tetramethylsilane as an internal standard. Column chromatography was accomplished either manually or on an Isco CombiFlash Separator System Sg100c. Thin layer chromatographic assessments were performed on silica gel. Elemental analyses were determined by Midwest Microlab LLC, Indianapolis, Indiana.

3-Hydroxy-4-phenyl[1,2,3] oxadiazolo[3,4-a]quinolin-10-ium inner salt (2a). Under an atmosphere of nitrogen, 3-[2-(phenylethynyl)phenyl]sydnone (1a) $(0.10 \mathrm{~g}, 0.38 \mathrm{mmol})$, was 
added to dry, conc $\mathrm{H}_{2} \mathrm{SO}_{4}(2 \mathrm{~mL})$ frozen at $-40{ }^{\circ} \mathrm{C}$. The flask was allowed to warm slowly to room temperature whereupon the reaction was poured carefully onto crushed ice $(\sim 15 \mathrm{~mL})$ and extracted with $\mathrm{CH}_{2} \mathrm{Cl}_{2}(3 \times 30 \mathrm{~mL})$. The combined extracts were dried $\left(\mathrm{MgSO}_{4}\right)$, filtered and the solvent removed in vacuo. Column chromatography (silica gel, $\mathrm{CH}_{2} \mathrm{Cl}_{2}$ ) gave a solid, which, upon recrystallization $\left(\mathrm{CH}_{2} \mathrm{Cl}_{2}\right.$ /hexanes) afforded the title compound as light yellow needles (0.012 g, 12\%), mp 250-251 ${ }^{\circ} \mathrm{C}$; IR (KBr): 3058 (w), 1742 (vs), 1722 (s), 1560 (m), 1497 (m), $748(\mathrm{~s}), 693(\mathrm{~m}) \mathrm{cm}^{-1} ;{ }^{1} \mathrm{H}$ NMR (DMSO- $\left.d_{6}, 100{ }^{\circ} \mathrm{C}\right): \delta 8.7-8.4(\mathrm{~m}, 9 \mathrm{H}) ;{ }^{13} \mathrm{C}$ NMR (DMSO- $d_{6}$, $\left.100{ }^{\circ} \mathrm{C}\right): 164.1(\mathrm{C}=\mathrm{O}), 134.0,133.5,132.0,130.6,129.9,129.8,129.5,129.1,128.7,127.5$, 121.9, 116.4 ppm. Elemental analysis: calculated for $\mathrm{C}_{16} \mathrm{H}_{10} \mathrm{~N}_{2} \mathrm{O}_{2}(262.26) \mathrm{C}: 73.27, \mathrm{H}: 3.84, \mathrm{~N}$ : 10.68. Found C: $72.99, \mathrm{H}: 3.83, \mathrm{~N}: 10.57 \%$.

General procedure for the synthesis of 3-arylcinnolines 3a-c from the corresponding orthoalkynylphenylsydnones 1a-c using trifluoromethanesulfonic acid. Freshly distilled $\mathrm{CF}_{3} \mathrm{SO}_{3} \mathrm{H}$ $(0.5 \mathrm{~mL}, 0.85 \mathrm{~g}, 0.006 \mathrm{~mol})$ was added dropwise to a solution of the ortho-alkynyl sydnone 1a-c $(0.10 \mathrm{~g})$ dissolved in $\mathrm{CH}_{2} \mathrm{Cl}_{2}(20 \mathrm{~mL})$. After $15 \mathrm{~min}$, the reaction was poured carefully onto crushed ice $(\sim 15 \mathrm{~mL})$, basified with $\mathrm{NaHCO}_{3}$, extracted with $\mathrm{CH}_{2} \mathrm{Cl}_{2}(3 \times 20 \mathrm{~mL})$, dried $\left(\mathrm{MgSO}_{4}\right)$ and the solvent removed in vacuo. Column chromatography on silica gel $\left(\mathrm{CH}_{2} \mathrm{Cl}_{2}\right.$ to $\mathrm{CH}_{2} \mathrm{Cl}_{2} / 1 \%$ THF as eluent), followed by recrystallization from $\mathrm{Et}_{2} \mathrm{O}$ or $\mathrm{CH}_{2} \mathrm{Cl}_{2} /$ hexanes, afforded 3a-c in $60-72 \%$ yield.

General procedure for the synthesis of 3-arylcinnolines 3d-f from the corresponding orthoalkynylphenylsydnones 1d-f using trifluoroacetic acid. The ortho-alkynylsydnone $1 \mathrm{~d}-\mathbf{f}(0.10$ g) was dissolved in $\mathrm{CF}_{3} \mathrm{CO}_{2} \mathrm{H}(5 \mathrm{~mL})$ and gently heated at reflux for 3-6 h. Subsequently, the reaction was cooled, diluted with $\mathrm{CH}_{2} \mathrm{Cl}_{2}(10 \mathrm{~mL})$, and evaporated to dryness in vacuo. The residue was taken up in $\mathrm{CH}_{2} \mathrm{Cl}_{2}(30 \mathrm{~mL})$, washed with saturated $\mathrm{NaHCO}_{3}$ solution $(3 \times 10 \mathrm{~mL})$, dried $\left(\mathrm{MgSO}_{4}\right)$ and the solvent removed in vacuo. Column chromatography (silica $\mathrm{gel}, \mathrm{CH}_{2} \mathrm{Cl}_{2}$ to $\mathrm{CH}_{2} \mathrm{Cl}_{2} / 1 \%$ THF as eluent), followed by recrystallization from $\mathrm{CH}_{2} \mathrm{Cl}_{2} /$ hexanes, $\mathrm{Et}_{2} \mathrm{O}$ or $\mathrm{Et}_{2} \mathrm{O} /$ hexanes afforded $\mathbf{3 d - f}$ in $77-86 \%$ yield.

3-(4-Pentylphenyl)cinnoline (3f). Reaction of 3-(2-(4-pentylphenylethynyl)phenyl)sydnone (1f) with $\mathrm{CF}_{3} \mathrm{CO}_{2} \mathrm{H}$ in the general procedure for $6 \mathrm{~h}$ gave after work-up yellow-orange crystals, $\mathrm{mp}$ 71-72 ${ }^{\circ} \mathrm{C}$; IR (KBr): 3046 (w), 2950 (m), 2921 (vs), 2849 (m), 1434 (m), 1322 (m), 1178 (m), $837(\mathrm{~m}), 746(\mathrm{~m}) \mathrm{cm}^{-1} ;{ }^{1} \mathrm{H} \mathrm{NMR}\left(\mathrm{CDCl}_{3}\right): \delta 8.53(\mathrm{~d}, J=9 \mathrm{~Hz}, 1 \mathrm{H}), 8.19-8.12(\mathrm{~m}, 3 \mathrm{H}), 7.85-7.68$ $(\mathrm{m}, 3 \mathrm{H}), 7.37(\mathrm{~d}, J=9 \mathrm{~Hz}, 2 \mathrm{H}), 2.70(\mathrm{t}, J=7.8 \mathrm{~Hz}, 2 \mathrm{H}), 1.71-1.66(\mathrm{~m}, 2 \mathrm{H}), 1.38-1.34(\mathrm{~m}, 2 \mathrm{H})$, 0.94-0.89 (m, 4H); ${ }^{13} \mathrm{C}-\mathrm{NMR}\left(\mathrm{CDCl}_{3}\right)$ : 153.8, 150.0, 144.8, 134.6, 131.4, 130.2, 130.1, 129.4, $127.4,127.2,126.7,118.5,36.0,31.8,31.3,22.8,14.3 \mathrm{ppm}$. Elemental analysis: calc. for $\mathrm{C}_{15} \mathrm{H}_{20} \mathrm{~N}_{2}$ (276.38) C: 82.57, H: 7.29, N: 10.14. Found C: 82.22, H: 7.21, N: $10.12 \%$.

\section{References}

1. Browne, D. L.; Harrity, J. P. A. Tetrahedron 2010, 66, 553. http://dx.doi.org/10.1016/j.tet.2009.10.085 
2. Earl, J. C.; Mackney, A. W. J. Chem. Soc 1935, 899.

http://dx.doi.org/10.1039/JR9350000899

3. Stewart, F. H. C. Chem. Rev. 1964, 64, 129.

http://dx.doi.org/10.1021/cr60228a004

4. Greco, C. V.; O'Reilly, B. P. J. Heterocycl. Chem. 1970, 7, 1433.

http://dx.doi.org/10.1002/jhet.5570070637

5. Greco, C. V.; O'Reilly, B. P. J. Heterocycl. Chem. 1972, 9, 207. http://dx.doi.org/10.1002/jhet.5570090207

6. Tien, H.-J.; Fang, G.-M.; Lin, S.-T.; Tien, L.-L. J. Chin. Chem. Soc. 1992, 39, 107. http://dx.doi.org/10.1002/jccs.199200017

7. Kalinin, V. N.; Min, S. F. J. Organomet. Chem. 1988, 352, C34. http://dx.doi.org/10.1016/0022-328X(88)83049-3

8. Burson, W. C., III; Jones, D. R.; Turnbull, K.; Preston, P. N. Synthesis 1991, 745. http://dx.doi.org/10.1055/s-1991-26564

9. Preston, P. N.; Turnbull, K. J. Chem. Soc., Perkin Trans. 1 1977, 1229.

10. Coburn, R. A.; O'Donnell, J. P. J. Org. Chem. 1972, 37, 1707. http://dx.doi.org/10.1021/jo00976a007

11. Lowe, J. D.; Turnbull, K. J. Heterocycl. Chem. 1986, 23, 125. http://dx.doi.org/10.1002/jhet.5570230125

12. Saljoughian, M.; Turnbull, K. J. Heterocycl. Chem. 1988, 25, 1817. http://dx.doi.org/10.1002/jhet.5570250641

13. Weisner, A. J.; Cooper, T. M.; Turnbull, K. Synth. Commun. 2005, 35, 671. http://dx.doi.org/10.1081/SCC-200050343

14. Baumgarten, H. E.; Furnas, J. L. J. Org. Chem. 1961, 26, 1536. http://dx.doi.org/10.1021/jo01064a056

15. Zhang, G.; Miao, J.; Zhao, Y.; Ge, H. Angew. Chem. Int. Ed. 2012, 51, 8318. http://dx.doi.org/10.1002/anie.201204339 PMid:22782712

16. Lowrie, H. S. J. Med. Chem. 1966, 9, 664. http://dx.doi.org/10.1021/jm00323a006

17. Ball, C. J.; Gilmore, J.; Willis, M. C. Angew. Chem. Int. Ed. 2012, 51, 5718. http://dx.doi.org/10.1002/anie.201201529 PMid:22539417

18. Gelvin, C. R.; Turnbull, K. Helv. Chim. Acta 1992, 75, 1931. http://dx.doi.org/10.1002/hlca.19920750619

19. Lewgowd, W.; Stanczak, A. Arch. Pharm. Chem. Life Sci. 2007, 340, 65. http://dx.doi.org/10.1002/ardp.200500194 PMid:17294399

20. von Richter, V. Chem. Ber. 1883, 16, 677. http://dx.doi.org/10.1002/cber.188301601154 
21. Alford, E. J.; Schofield, K. J. Chem. Soc. 1952, 2102. http://dx.doi.org/10.1039/jr9520002102

22. Baumgarten, H. E.; Anderson, C. H. J. Amer. Chem. Soc. 1958, 80, 1981. http://dx.doi.org/10.1021/ja01541a050

23. Gomaa, M. A. M. Tetrahedron Lett. 2003, 44, 3493. http://dx.doi.org/10.1016/S0040-4039(03)00686-5

24. Chen, D.; Yang, C.; Xie, Y.; Ding, J. Heterocycles 2009, 77, 273. http://dx.doi.org/10.3987/COM-08-S(F)7

25. Vinogradova, O. V.; Balova, I. A. Chem. Heterocycl. Compd. 2008, 44, 501. http://dx.doi.org/10.1007/s10593-008-0070-0 